\title{
Effectiveness of Oral Health Education Program on Prevention of Periodontal Disease in Japanese Pregnant Women
}

\author{
Mayumi Noguchi'1,2, Akira Tagaya ${ }^{3}$, Ayako Sakoda' ${ }^{1}$, Hitoshi Komatsuzawa ${ }^{4,5}$, \\ Natsumi Fujiwara ${ }^{4,6}$, Motoyuki Sugai ${ }^{4}$ \\ ${ }^{1}$ Japanese Red Cross Hiroshima College of Nursing, Hatsukaichi, Japan \\ 2Japanese Red Cross Toyota College of Nursing, Toyota, Japan (Current) \\ ${ }^{3}$ Nagano College of Nursing, Komagane, Japan \\ ${ }^{4}$ Department of Bacteriology, Hiroshima University Graduate School of Biomedical \& Health Sciences, \\ Hiroshima, Japan \\ ${ }^{5}$ Department of Oral Microbiology, Kagoshima University Graduate School of Medical and Dental Sciences, \\ Kagoshima, Japan (Current) \\ ${ }^{6}$ Department of Oral Health Care Promotion, Tokushima University Graduate School of Biomedical Sciences, \\ Tokushima, Japan (Current) \\ Email: noguchi@rctoyota.ac.jp
}

Received 6 March 2016; accepted 16 April 2016; published 19 April 2016

Copyright (C) 2016 by authors and Scientific Research Publishing Inc.

This work is licensed under the Creative Commons Attribution International License (CC BY). http://creativecommons.org/licenses/by/4.0/

(c) (i) Open Access

\begin{abstract}
The prevalence of periodontal disease among pregnant women increases with gestational age. Therefore, oral health education in the early stage of pregnancy should prevent periodontal disease and decrease the risk of an adverse pregnancy outcome such as preterm delivery and low birth weight. However, there has been no study on oral health care intervention for prevention of periodontal disease during pregnancy. The purpose of this study was to examine the efficiency of an oral health education program on periodontal disease among Japanese low-risk pregnant women. A total of 207 pregnant women before 20 weeks of gestation were recruited. The first 131 of them were assigned to the control group and the remaining 76 to the experimental group. The experiment participants received an oral health education program including the toothpick brushing method in their early stage of pregnancy. Improvement of the self-assessment score of periodontal symptoms was used as a binary outcome variable. A logistic regression analysis indicated that the intervention $(O R=3.83)$ and proficiency of the toothpick brushing method $(O R=$ 24.93) were statistically significant predictors of the outcome in the late stage of pregnancy. The positive outcome appeared after practice of the toothpick method brushing for 20 or more weeks, and was associated with the decrease in the log-transformed proportion of the count of Candida species in salivary microbiota $(p=0.026)$.
\end{abstract}




\section{Keywords}

\section{Periodontal Disease, Pregnancy, Oral Health, Educational Intervention, Toothpick Brushing Method}

\section{Introduction}

Prevalence of gingival inflammation increases in pregnancy and the increased plasma levels of estrogen and progesterone are believed to be responsible for this poorer oral health status although its mechanism is not fully revealed [1]. Vogt et al. reported that the prevalence of periodontal disease (PD) increased with gestation age $[2]$.

An oral health education program in the early stage of pregnancy will help pregnant women keep their oral health throughout their pregnancy. American College of Obstetricians and Gynecologists, Committee on Health Care for Underserved Women recommends oral health care during pregnancy [3]. However, many people wrongly believe that decreased dental health is unavoidable during pregnancy [4] and only a few women receive dental health checkup during pregnancy. It is necessary to provide them with oral health education to prevent PD. However, there has been no study on such an intervention for prevention of PD during pregnancy.

Moreover, it is very likely that PD is a risk factor for preterm birth and low birth weight (LBW) according to the case-control study by Offenbacher et al. [5] and the prospective study by Jeffcoat et al. [6]. Although it is not clear whether the treatment of PD during pregnancy is effective in preventing preterm birth or LBW [7]-[9], it is very likely that the risk of preterm birth and LBW can be lowered by prevention of PD.

The purpose of this study was to examine the effectiveness of an oral health education program on the selfassessed status of oral health related to PD.

\section{Methods}

\subsection{Research Design}

Our study was based on the assumption that the status of oral health related to PD is affected by oral health and other lifestyle behaviors and psychological status. A quasi-experimental research design was adopted to avoid contact between the experiment and control participants because the research was carried out at one hospital. The period of data collection was from September of 2007 to March of 2010. In the beginning the participants were placed in the control group and then later others were assigned to the experimental group.

\subsection{Inclusion Criteria}

We recruited healthy pregnant women before 20 weeks of singleton pregnancy without medical history of diabetes or hypertension.

\subsection{Data Collection and Intervention}

The questionnaire responses and oral microbial materials were collected by an author (M. N. or A. S.) and a dental hygienist when they met the participants three times during pregnancy. The experimental group participants received an educational program at the time of the first survey, and received a plaque checks four or more weeks after the first and second surveys.

The gestational age of the subjects was 8 - 19 weeks (hereafter designated as T1) for the first survey, 20 - 29 weeks (T2) for the second survey, and 30 - 38 weeks (T3) for the third survey, with four or more weeks intervals. There were a few exceptions allowing up to three weeks of deviation for the convenience of participants (Table 1).

\subsection{Questionnaire}

The following scales were used to assess the factors that would affect the state of oral health related to PD. 
Table 1. Schedule of data collection and intervention. The intervention included an oral health education, toothpick-method brushing training, and plaque checks with assessment of OHI-S.

\begin{tabular}{cccc}
\hline \multicolumn{1}{c}{ Activity } & Gestational age & Data collected & Intervention \\
Baseline survey & $8-19$ weeks $(=\mathrm{T} 1)$ & $\begin{array}{l}\text { QD at T0 } \\
\text { QD at T1 } \\
\text { OB at T1 }\end{array}$ & $\begin{array}{c}\text { Show an educational video for prevention of PD and } \\
\text { teach toothpick brushing method using a dental model }\end{array}$ \\
Plaque check \#1 & 4 or more weeks after T1 & OHI-S \#1 & Show stained plaque and correct brushing \\
Intermediate survey & 20 - 29 weeks (=T2) & $\begin{array}{l}\text { QD at T2 } \\
\text { OB at T2 }\end{array}$ & Consultation if necessary \\
Plaque check \#2 & 4 or more weeks after T2 & OHI-S \#2 & Show stained plaque and correct brushing \\
Final survey & 30 - 38 weeks (=T3) & OB at T3 & Consultation if necessary
\end{tabular}

QD: questionnaire data; T0: before pregnancy; OB: oral bacteria; OHI-S: Simplified Oral Hygiene Index.

\subsubsection{The Self-Assessment of Periodontal Disease (SAPD)}

This scale developed by Nakamura et al. consists of six questions about subjective evaluation (each on a four-grade Likert scale) of symptoms of PD, such as gingival bleeding, subgingival abscess, and gingivalgia. Its discriminating validity was checked with diagnoses based on Community Periodontal Index (CPI) using multiple logistic regression analysis. The predictive accuracy depended on age: $71 \%$ for $20 \mathrm{~s}, 75 \%-77 \%$ for $30 \mathrm{~s}$, and $80 \%-82 \%$ for $40 \mathrm{~s}$, which reflects the fact that severe PD increases from $30 \mathrm{~s}$ [10].

We used SAPD to evaluate the effect of the intervention. As the outcome variable, we used a binary variable representing improvement of SAPD (whether the score decreased or not from the score before pregnancy) at T2 and T3 instead of SAPD itself to best avoid the effects of individual tendencies in rating and the difference at the baseline.

\subsubsection{Oral Hygiene Behaviors}

Oral hygiene behaviors were assessed by the frequency and duration of tooth brushing, the use of mouthrinse, and frequency of scaling. Subjective rating of sufficiency of oral health education they had received to date was also asked at $\mathrm{T} 3$.

\subsubsection{Lifestyle Habits/Psychological State}

The questionnaire included questions about lifestyle habits: eating (20 items), sleeping (4 items), physical exercise $(1$ item), drinking $(1$ item), and smoking ( 1 item). Also, the psychological state was measured using the Japanese version of General Health Questionnaire (GHQ 28) [11].

\subsection{Microbial Counts}

The status of the microbial environment in the mouth was assessed by culture methods followed by identification of microorganism by PCR. The total cultivable microbial count, prevalence of Candida species and Staphylococcus aureus, and the proportion of counts of Candida species and S. aureus to the total cultivable microbial count were used for analysis of association of microbiota with the outcome. The methods of culture and PCR are described in Fujiwara et al. [12]. For count $n, \log (n+1)$ was used in data analyses.

\subsection{Oral Health Education Program}

Following the data collection at T1, experiment participants received an oral health education using a DVD displayer and training of the toothpick brushing method by a dental hygienist. They received plaque checks four or more weeks after T1 and T2. Consultation regarding tooth brushing was provided on request after data collection at T2 and T3. No restriction was made about additional oral health activities, and the frequencies of use of dental floss, mouthrinse, and professional plaque control during the intervention period were asked in the questionnaire. 


\subsubsection{Oral Health Education Using a DVD Player}

A 15-minute program for prevention of PD provided the experiment participants with the knowledge for regular plaque control, smoking cessation, obesity prevention, relieving stress, and physical exercise.

\subsubsection{Training of Tooth Brushing}

For tooth brushing in intervention we employed the toothpick method, which was designed to remove dental plaque and relieve inflammation in interdental area, and has been proved to be effective with self-brushing by healthy dental students [13], professional brushing for malodor periodontal patients [14], and an educational intervention for patients with mental disorders [15].

The following directions were used in training experiment participants for toothpick method by a dental hygienist and illustrated by photographs using a plastic model in the leaflet given to the trainees.

1) Place the tips of the bristles on the gingival margin toward the crown at an angle of 30 degrees on the vestibular surface and vertically on the lingual surface.

2) Push the bristles into the interdental area and then pulled them out. Repeat this stroke seven or eight times in each interdental area.

The participants used a toothbrush with two rows of nylon bristles, five tufts per row, and 50 filaments per tuft specially designed for the toothpick method (PMJ V7).

\subsubsection{Plaque Check}

Simplified Oral Hygiene Index (OHI-S) [16] was employed to assess the status of oral hygiene.

\subsection{Method of Analysis}

The effects of intervention and other factors on the outcome variable were examined for statistical significance by cross tables and logistic regression analysis (LRA). The relation of the outcome with microbial data was examined by Mann-Whitney's U test and cross tables. The statistical software package SPSS (version 22) was used for these analyses.

\subsection{Ethical Consideration}

This research was approved by the IRB of Japanese Red Cross Hiroshima College of Nursing. The subjects were those who provided written consent to participate in the study after a process of informed consent using both oral and written explanation. The saliva collection, tooth-brushing education, and plaque check were carried out in a room for privacy.

\section{Results}

\subsection{Participants}

A total of 207 participants were recruited. The first 131 of them were assigned to the control group and the rest to the experimental group $(n=76)$. Table 2 shows their obstetric history about parity and the outcome of the pregnancy. The proportion of multipara was higher in the control group although the difference was not statistically significant at $5 \%$ level. The age, stature, body weight, gestational age, and birth weight are shown in Table 3 , where no statistical significant difference was detected between the two groups. There was no statistically significant group difference in lifestyle habits (sleeping, eating, drinking, and smoking), oral health behaviors (tooth brushing, rinsing, flossing, and dental health check), except that the control group enjoyed sports more frequently (chi2 $=4.544$, d.f. $=1, p=0.048)$ and had higher average of GHQ score $(p=0.002)$.

\subsection{Measurements and Interventions}

The data were collected from each participant at three scheduled times by questionnaires and salivary samplings. These times will be designated as $\mathrm{T} 1, \mathrm{~T} 2$, and $\mathrm{T} 3$, and $\mathrm{T} 0$ designates the time period just before pregnancy. The questionnaire at $\mathrm{T} 1$ included the responses to questions about the statuses at $\mathrm{T} 0$ and $\mathrm{T} 1$. The results of dental plaque checks (OHI-S) four or more weeks after T1 and T2 will be designated as OHI-S \#1 and OHI-S \#2.

Table 4 shows rate of attendance of participants in each survey. The attendance rate did not differ between groups at T2 (exact test, $p=0.443$ ). At T3, however, the attendance rate in the experimental group $(61.8 \%$ ) was 
Table 2. Parity and outcome of the pregnancy of the participants.

\begin{tabular}{|c|c|c|c|c|c|}
\hline \multirow{2}{*}{ Classification } & \multicolumn{2}{|c|}{ Experimental group } & \multicolumn{2}{|c|}{ Control group } & \multirow{2}{*}{$\begin{array}{c}\text { Group difference } \\
\text { (exact test) }\end{array}$} \\
\hline & $\mathrm{n}$ & (\%) & $\mathrm{n}$ & (\%) & \\
\hline \multicolumn{6}{|l|}{ (Parity) } \\
\hline Primipara & 37 & $(48.7)$ & 47 & (35.9) & \\
\hline Multipara & 37 & $(48.7)$ & 79 & $(60.3)$ & $p=0.102$ \\
\hline Unknown & 2 & (2.6) & 5 & $(3.8)$ & \\
\hline \multicolumn{6}{|l|}{ (Outcome of pregnancy) } \\
\hline Normal delivery & 60 & $(78.9)$ & 99 & $(75.6)$ & \\
\hline Abortion/Premature delivery & 3 & (3.9) & 9 & (6.9) & $p=0.538$ \\
\hline Unknown (hospital transfer) & 13 & $(17.1)$ & 23 & $(17.6)$ & \\
\hline Total & 76 & (100.0) & 131 & (100.0) & \\
\hline
\end{tabular}

Table 3. Physical attributes of participants, gestational age at delivery, and birthweight.

\begin{tabular}{|c|c|c|c|c|c|c|c|c|c|c|}
\hline \multirow{2}{*}{$\begin{array}{l}\text { Attribute of } \\
\text { participants }\end{array}$} & \multicolumn{4}{|c|}{ Experimental group } & \multicolumn{4}{|c|}{ Control group } & \multicolumn{2}{|c|}{ Group difference } \\
\hline & $\mathrm{n}$ & Mean & SD & Range & $\mathrm{n}$ & Mean & SD & Range & t-value & $p$ \\
\hline Age & 74 & 29.3 & 5.0 & $17-38$ & 127 & 29.3 & 5.6 & $17-45$ & -0.082 & 0.935 \\
\hline Stature (cm) & 74 & 157.2 & 5.0 & $148-170$ & 127 & 157.7 & 5.4 & $145-173$ & 0.549 & 0.584 \\
\hline Body weight* (kg) & 72 & 51.7 & 9.1 & $37-96$ & 124 & 51.9 & 9.0 & $37-98$ & 0.197 & 0.844 \\
\hline Delivery weeks & 62 & 40.9 & 1.3 & $38-43$ & 101 & 41.1 & 1.3 & $37-43$ & 0.752 & 0.453 \\
\hline Birth weight (kg) & 62 & 3.01 & 0.34 & $2.37-3.89$ & 102 & 3.00 & 0.36 & $2.04-4.02$ & -0.101 & 0.920 \\
\hline
\end{tabular}

*body weight before pregnancy answered at baseline survey.

Table 4. Sample size and gestational age of subjects participated in the surveys. The percentage after sample size $\mathrm{n}$ indicates the rate of attendance of participants.

\begin{tabular}{|c|c|c|c|c|c|c|c|c|c|c|c|}
\hline \multirow{3}{*}{ Data collection } & \multicolumn{4}{|c|}{ Experimental group } & \multicolumn{4}{|c|}{ Control group } & \multirow{2}{*}{\multicolumn{3}{|c|}{$\begin{array}{l}\text { Group difference } \\
\text { (Mann-Whitney) }\end{array}$}} \\
\hline & \multirow{2}{*}{ n (\%) } & \multicolumn{3}{|c|}{ Gestational week } & \multirow{2}{*}{ n (\%) } & \multicolumn{3}{|c|}{ Gestational week } & & & \\
\hline & & Mean & SD & Range & & Mean & SD & Range & $\mathrm{U}$ & $\mathrm{z}$ & $p$ \\
\hline Baseline survey & $76(100)$ & 12.7 & 2.1 & $8-20$ & $131(100)$ & 12.8 & 2.3 & 817 & 4858.0 & -0.293 & 0.770 \\
\hline Plaque check \#1 & $59(77.6)$ & 18.0 & 2.8 & $13-27$ & & & & & & & \\
\hline Intermediate survey & $56(73.7)$ & 23.1 & 2.9 & $19-32$ & 99 (75.6) & 23.0 & 2.1 & $20-29$ & 2666.5 & -0.399 & 0.690 \\
\hline Plaque check \#2 & $49(64.5)$ & 26.9 & 2.8 & $23-36$ & & & & & & & \\
\hline Final survey & 47 (61.8) & 33.1 & 2.8 & $27-40$ & $98(74.8)$ & 34.2 & 1.7 & $29-40$ & 1657.5 & -2.779 & 0.005 \\
\hline Perfect attendance & $46(60.5)$ & & & & 87 (66.4) & & & & & & \\
\hline
\end{tabular}

lower than in the control group (74.8\%) with statistical significance (exact test, $p=0.035$ ) due to the group difference in the rate of attendance at T3 among the participants who were absent at T2; they were 35.3\% (12/34) in the control group and $4.8 \%(1 / 21)$ in the experimental group (exact test, $p=0.017$ ). There was no statistically significant group difference in the final perfect attendant rate: 66.4\% (87/131) in the control group and 60.5\% (46/76) in the experimental group (exact test, $p=0.452$ ).

The gestational age did not differ between groups for T1 and T2, but that of T3 was somewhat earlier in the 
experimental group (33.1 \pm 2.8 weeks) than in the control group (34.2 \pm 1.7 weeks). This resulted from setting the date in accordance with the convenience of participants (Table 4).

Since the gestational age of T3 was not statistically associated with improvement of SAPD, the effect must be very limited. Theoretically, however, the effect is not negligible as the outcome could change between T2 and T3. Therefore, the gestational age at T3 was included among the possible predictors for stepwise selection in LRA.

\subsection{Subjective Rating of Periodontal Symptoms}

SAPD0, SAPD1, SAPD2, and SAPD3 shall denote the scores of SAPD at T0, T1, T2, and T3. Imp2 and Imp3 shall denote the improvement of periodontal symptoms at T2 and T3. Thus, if SAPD2 < SAPD0 then Imp2 = 1 (positive outcome) else $\operatorname{Imp} 2=0$ (non-positive outcome), and if SAPD3 < SAPD0 then Imp3 = 1 (positive outcome) else Imp3 = 0 (non-positive outcome).

The group difference on the rate of positive outcome was examined to evaluate the effect of intervention at T2 and T3 (Table 5). The rate of positive outcome was almost the same (22\% and 23\%) for the two groups at T2. At T3, however, it nearly doubled in the experimental group while it slightly decreased in the control group. The exact test of cross tables indicated that no substantial effect of intervention emerged at T2, and a positive effect emerged at T3. The OR of this positive outcome (at T3) for our educational intervention was compared between those who showed improvement of SAPD at T2 and the rest (Table 6). In both of them the intervention yielded similar ORs (3.11 vs 3.57) and the Breslow-Day's test indicated that the effect of intervention on the outcome at T3 did not depend on the status of periodontal symptoms at T2, which validated the Mantel-Haenszel's estimation of common OR (=3.44).

Table 5. The outcome of intervention at T2 and T3 expressed by existence of improvement of the status of periodontal symptoms compared to the status before pregnancy.

\begin{tabular}{|c|c|c|c|c|c|c|c|c|c|c|}
\hline \multirow{2}{*}{ Time } & \multirow{2}{*}{\multicolumn{2}{|c|}{ Outcome }} & \multicolumn{2}{|c|}{ Experimental group } & \multicolumn{2}{|c|}{ Control group } & \multicolumn{2}{|c|}{ Total } & \multirow{2}{*}{$\begin{array}{l}\text { Group difference } \\
\quad \text { (exact test) }\end{array}$} & \multirow{2}{*}{ OR } \\
\hline & & & $\mathrm{n}$ & $(\%)$ & $\mathrm{n}$ & (\%) & $\mathrm{n}$ & (\%) & & \\
\hline \multirow{3}{*}{$\mathrm{T} 2$} & Positive & $(\operatorname{Imp} 2=1)$ & 12 & $(22.2)$ & 21 & (23.3) & 33 & (22.9) & \multirow{3}{*}{$p=1.000$} & \multirow{3}{*}{0.94} \\
\hline & Non-positive & $(\operatorname{Imp} 2=0)$ & 42 & (77.8) & 69 & $(76.7)$ & 111 & $(77.1)$ & & \\
\hline & Total & & 54 & $(100.0)$ & 90 & $(100.0)$ & 144 & $(100.0)$ & & \\
\hline \multirow{3}{*}{$\mathrm{T} 3$} & Positive & $(\operatorname{Imp} 3=1)$ & 19 & $(43.2)$ & 20 & $(23.0)$ & 39 & $(29.3)$ & \multirow{3}{*}{$p=0.025$} & \multirow{3}{*}{2.53} \\
\hline & Non-positive & $(\operatorname{Imp} 3=0)$ & 25 & $(56.8)$ & 67 & $(77.0)$ & 94 & (70.7) & & \\
\hline & Total & & 44 & $(100.0)$ & 87 & $(100.0)$ & 133 & $(100.0)$ & & \\
\hline
\end{tabular}

Table 6. The effect of intervention on the outcome at T3 (Imp3) assessed by OR and examination of its dependence on the status at T2 (Imp2).

\begin{tabular}{|c|c|c|c|c|c|c|c|}
\hline \multirow[b]{2}{*}{ Outcome at T2 } & \multirow[b]{2}{*}{ Group } & \multicolumn{4}{|c|}{ Outcome at T3 } & \multirow[b]{2}{*}{ OR } & \multirow{2}{*}{$\begin{array}{l}\text { Group difference } \\
\text { (exact test) }\end{array}$} \\
\hline & & $\begin{array}{c}\text { Positive } \\
(\text { Imp3 = 1) }\end{array}$ & $\begin{array}{l}\text { Non-positive } \\
(\operatorname{Imp3}=0)\end{array}$ & Subtotal & Positive \% & & \\
\hline \multirow{3}{*}{$\begin{array}{c}\text { Positive } \\
\text { (Imp2 = 1) }\end{array}$} & Experimental & 8 & 2 & 10 & 80.0 & \multirow{3}{*}{3.11} & \multirow{3}{*}{$p=0.399$} \\
\hline & Control & 9 & 7 & 16 & 56.3 & & \\
\hline & Subtotal & 17 & 9 & 26 & 65.4 & & \\
\hline \multirow{3}{*}{$\begin{array}{l}\text { Non-positive } \\
(\operatorname{Imp} 2=0)\end{array}$} & Experimental & 11 & 22 & 33 & 33.3 & \multirow{3}{*}{3.57} & \multirow{3}{*}{$p=0.027$} \\
\hline & Control & 7 & 50 & 57 & 12.3 & & \\
\hline & Subtotal & 18 & 72 & 90 & 20.0 & & \\
\hline \multirow{3}{*}{ Total } & Experimental & 19 & 24 & 43 & 44.2 & \multirow{3}{*}{2.82} & \multirow{3}{*}{$p=0.020$} \\
\hline & Control & 16 & 57 & 73 & 21.9 & & \\
\hline & Subtotal & 35 & 81 & 116 & 30.2 & & \\
\hline \multicolumn{4}{|c|}{ Dependence of OR on Imp2 (Breslow-Day’s test) } & \multicolumn{4}{|c|}{ Effect of intervention (Mantel-Haenszel’s estimation) } \\
\hline Chi-square & \multicolumn{2}{|c|}{ Probability } & \multicolumn{3}{|c|}{ Common OR of positive outcome } & \multicolumn{2}{|c|}{$95 \%$ CI of common OR } \\
\hline 0.016 & \multicolumn{2}{|c|}{0.899} & \multicolumn{3}{|c|}{3.44} & \multicolumn{2}{|r|}{$1.36-8.70$} \\
\hline
\end{tabular}




\subsection{LRA of Binary Outcome}

The variables that exhibited statistically significant group difference and parity were included in the LRA to predict binary outcome $\operatorname{Imp} 2$ and $\operatorname{Imp} 3$. The parity was unconditionally included and others were subject to selection by the variable reduction method. First, the whole sample was analyzed where the group assignment was included as a predictor. Second, the data of the experimental group was analyzed.

\subsubsection{LRA of Outcome at T2 (=Imp2)}

Affirmative rating of sufficiency of oral health education and regular scaling before pregnancy contributed to positive outcome (Imp2 $=1$, i.e., SAPD2 < SAPD0) with statistical significance (Table 7). The omnibus test proved statistical significance of the model coefficients (chi2 $=10.203$, d.f. $=3, p=0.017$ ). The Hosmer $\&$ Lemeshow's test indicated a good fit of the logistic regression model obtained (chi2 $=0.381$, d.f. $=5, p=0.996$ ).

The $\exp (\mathrm{B})$ in the table, which is interpreted as the odds ratio of positive outcome $(\operatorname{Imp} 2=1)$ with other predictors unchanged, was 3.03 for sufficient oral health education and 2.68 for regular scaling before pregnancy. The odds ratio of positive outcome for multiparity was 1.76 although not statistically significant.

The univariate odds ratio of positive outcome $(\operatorname{Imp} 2=1)$ was 2.89 for sufficient oral health education, 2.78 for regular scaling, and 1.60 for multiparity.

\subsubsection{LRA of Outcome at T3 (=Imp3)}

The statistically significant predictors were $\operatorname{Imp} 2$, mouthrinse at T1, being assigned to experimental group, and regular scaling before pregnancy (Table 8). The omnibus test of the coefficients indicated statistical significance (chi2 $=39.764$, d.f. $=5, p=1.7 \mathrm{E}-7$ ), and the Hosmer $\&$ Lemeshow's test indicated a sufficient fit of the model obtained (chi2 $=7.306$, d.f. $=6, p=0.293$ ). The odds ratio of positive outcome $(\operatorname{Imp} 3=1$, i.e., SAPD3 < SAPD0) expressed by $\exp (\mathrm{B})$ in the table was 9.92 for $\operatorname{Imp} 2,6.08$ for mouthrinse at $\mathrm{T} 1,3.83$ for assignment to experimental group, and 3.35 for regular scaling before pregnancy. The odds ratio of multiparity was 1.59 although not statistically significant.

The univariate odds ratio was 7.56 for Imp2, 3.68 for mouthrinse at T1, 2.55 for assignment to experimental group, and 1.99 for regular scaling before pregnancy. The univariate odds ratio of parity was 1.68.

Table 7. Logistic regression analysis of Imp2 (outcome at T2).

\begin{tabular}{ccccccccc}
\hline Predictor variable & \multirow{2}{*}{ B } & S.E. & Wald & $p$ & exp $(\mathrm{B})$ & \multicolumn{2}{c}{$95 \% \mathrm{CI}$ of $\exp (\mathrm{B})$} \\
\cline { 6 - 9 } & & & & & & L. L. & U. L. \\
\hline Sufficient oral health education & 1.110 & 0.551 & 4.05 & 0.044 & 3.03 & 1.03 & 8.94 \\
Regular scaling before pregnancy & 0.987 & 0.502 & 3.86 & 0.049 & 2.68 & 1.00 & 7.18 & 4.29 \\
Multipara & 0.564 & 0.456 & 1.53 & 0.215 & 1.76 & 0.72 & \\
(Constant) & -2.494 & 0.584 & 18.22 & $<0.001$ & 0.08 & & \\
\hline
\end{tabular}

L.L. \& U.L. = Lower \& Upper Limits.

Table 8. Logistic regression analysis of Imp3 (outcome at T3).

\begin{tabular}{|c|c|c|c|c|c|c|c|}
\hline \multirow{2}{*}{ Predictor variable } & \multirow{2}{*}{ B } & \multirow{2}{*}{ S.E. } & \multirow{2}{*}{ Wald } & \multirow{2}{*}{$p$} & \multirow{2}{*}{$\exp (\mathrm{B})$} & \multicolumn{2}{|c|}{$95 \%$ CI of $\exp (\mathrm{B})$} \\
\hline & & & & & & L. L. & U. L. \\
\hline Imp2 (positive outcome at T2) & 2.294 & 0.572 & 16.09 & $6.0 \mathrm{E}-5$ & 9.92 & 3.23 & 30.43 \\
\hline Mouthrinse at T1 & 1.805 & 0.590 & 9.35 & 0.002 & 6.08 & 1.91 & 19.32 \\
\hline Assigned to experimental group & 1.344 & 0.522 & 6.64 & 0.010 & 3.83 & 1.38 & 10.66 \\
\hline Regular scaling before pregnancy & 1.210 & 0.590 & 4.20 & 0.040 & 3.35 & 1.05 & 10.67 \\
\hline Multipara & 0.465 & 0.512 & 0.83 & 0.363 & 1.59 & 0.58 & 4.35 \\
\hline (Constant) & -4.309 & 1.001 & 18.54 & $1.7 \mathrm{E}-5$ & 0.01 & & \\
\hline
\end{tabular}

L.L. \& U.L. = Lower \& Upper Limits. 


\subsubsection{LRA of Outcome at T2 in Experimental Group}

An LRA of Imp2 was carried out using the dataset including the variables related to the intervention. The sample size was 50 . As shown in Table 9, only the regular scaling before pregnancy was statistically significant predictor. The omnibus test indicated the tendency toward statistical significance of the model (chi2 $=4.799$, d.f. $=2$, $p=0.091$ ) and the Hosmer $\&$ Lemeshow's test indicated a good fitting of the model (chi2 $=0.467$, d.f. $=2, p=$ $0.792)$.

The univariate odds ratio for regular scaling before pregnancy was 5.29 , which is almost equal to $\exp (\mathrm{B})$ ( = 5.28).

\subsubsection{LRA of Outcome at T3 in Experimental Group}

An LRA of Imp3 was carried out using the dataset including the variables related to the intervention. Although the sample size was small $(\mathrm{n}=39)$, the omnibus test indicated statistical significance of the model (chi2 $=$ 19.475, d.f. $=4, p=0.001$ ). The Hosmer $\&$ Lemeshow's test indicated a good fit of the model (chi2 $=1.368$, d.f. $=5, p=0.928)$. As shown in Table 10, proficiency of toothpick method brushing (OR $=24.93)$, mouthrinse at $\mathrm{T} 1$ (OR $=13.56)$, and $\operatorname{Imp} 2(\mathrm{OR}=13.08)$ were statistically significant predictors, and the contribution of multiparity $(\mathrm{OR}=5.75)$ approached statistical significance $(p=0.091)$.

The univariate odds ratio was 5.45 for proficiency of toothpick method brushing, 3.38 for mouthrinse at T1, 8.00 for $\operatorname{Imp} 2$, and 1.75 for multiparity. The difference from the multivariate results can be explained by the negative correlations within these variables.

\subsubsection{Brushing Proficiency and Dental Plaque}

The score of OHI-S was compared between those who rated affirmatively and those who rated negatively about their proficiency of toothpick method brushing. Mann-Whitney's U indicated that those who highly rated their proficiency had lower plaque score in both the comparisons of OHI-S \#1 (one sided $p=0.037$ ) and OHI-S \#2 (one sided $p=0.024$ ).

\subsubsection{Relation of Microbiota with the Outcome}

The total cultivable microbial count exhibited no statistically significant association with $\operatorname{Imp} 2$ and $\operatorname{Imp} 3$ in both groups. The proportion of Candida count at T1 was positively associated with $\operatorname{Imp} 2(p=0.005)$ and $\operatorname{Imp} 3(p=$ 0.009) only in the experimental group, while that of Staphylococci exhibited no such difference.

Table 9. Logistic regression analysis of Imp2 (outcome at T2) in experimental group.

\begin{tabular}{|c|c|c|c|c|c|c|c|}
\hline \multirow{2}{*}{ Predictor variable } & \multirow{2}{*}{ B } & \multirow{2}{*}{ S.E. } & \multirow{2}{*}{ Wald } & \multirow{2}{*}{$p$} & \multirow{2}{*}{$\exp (\mathrm{B})$} & \multicolumn{2}{|c|}{$95 \%$ CI of $\exp (\mathrm{B})$} \\
\hline & & & & & & L. L. & U. L. \\
\hline Regular scaling before pregnancy & 1.664 & 0.755 & 4.86 & 0.028 & 5.28 & 1.20 & 23.19 \\
\hline Multipara & 0.076 & 0.691 & 0.01 & 0.912 & 1.08 & 0.28 & 4.18 \\
\hline (Constant) & -1.702 & 0.533 & 10.18 & 0.001 & 0.18 & & \\
\hline
\end{tabular}

L.L. \& U.L. = Lower \& Upper Limits.

Table 10. Logistic regression analysis of Imp3 (outcome at T3) in experimental group.

\begin{tabular}{|c|c|c|c|c|c|c|c|}
\hline \multirow{2}{*}{ Predictor variable } & \multirow{2}{*}{ B } & \multirow{2}{*}{ S.E. } & \multirow{2}{*}{ Wald } & \multirow{2}{*}{$p$} & \multirow{2}{*}{$\exp (\mathrm{B})$} & \multicolumn{2}{|c|}{$95 \%$ CI of $\exp (\mathrm{B})$} \\
\hline & & & & & & L. L. & U. L. \\
\hline Brushing proficiency at T2 & 3.216 & 1.322 & 5.92 & 0.015 & 24.93 & 1.87 & 332.46 \\
\hline Mouthrinse used at T1 & 2.607 & 1.145 & 5.18 & 0.023 & 13.56 & 1.44 & 128.97 \\
\hline Imp2 (positive outcome at T2) & 2.571 & 1.156 & 4.95 & 0.026 & 13.08 & 1.36 & 126.00 \\
\hline Multipara & 1.750 & 1.034 & 2.86 & 0.091 & 5.75 & 0.76 & 43.68 \\
\hline (Constant) & -3.013 & 1.073 & 7.89 & 0.005 & 0.05 & & \\
\hline
\end{tabular}

L.L. \& U.L. = Lower \& Upper Limits. 
The prevalence of Candida species was 38.2\% (78/204) at T1, 41.1\% (62/151) at T2, and 44.3\% (62/140) at T3 without statistically significant group difference. Interestingly, the rate of positive outcome among Candi$d a$-positive subjects exhibited a significant group difference at both $\mathrm{T} 2$ and $\mathrm{T} 3$. Their rate of positive outcome at T2 was 38.9\% (7/18) in the experimental group which was significantly greater than $12.8 \%$ ( $5 / 39$ ) in the control group (exact test, $p=0.037)$. Their rate of positive outcome at T3 was $50.0 \%(8 / 16)$ in experimental group which was significantly greater than $19.4 \%(7 / 36)$ in the control group (exact test, $p=0.044$ ). The Candida-negative participants exhibited no such group difference at T2 and T3. In other words, the effect of "assignment to the experimental group” on the outcome at T2 and T3 was statistically significant only for the subjects who were Candida-positive at T1.

Imp3 was associated with neither the prevalence of Candida nor the proportion of Candida count at T3 in both groups, but positively associated with the decrease in the proportion of Candida count from T1 to T3 (Spearman's $\rho=0.203, p=0.025$ ).

The prevalence of Staphylococcus aureus was as high as $90.6 \%(186 / 203)$ at T1, 86.2\% (131/152) at T2, and 93.5\% (129/138) at T3 without statistically significant group difference. It was not possible to examine the association of its prevalence with the outcome because most of the subjects possessed $S$. aureus.

\section{Discussion}

\subsection{Efficiency of Intervention}

Improvement of SAPD score was used as the binary outcome variable, and its LRAs indicated that the intervention $(\mathrm{OR}=3.83)$ and brushing proficiency $(\mathrm{OR}=24.93)$ significantly contributed to predict the outcome at T3.

As Vogt et al. [2] demonstrated using a set of sound criteria for diagnosis, the prevalence of PD increases with gestation age even in low-risk pregnant women. The efficiency of the toothpick method had not been examined for pregnant women. Our results showed, however, that the educational intervention and resulting proficiency of toothpick method brushing could prevent exacerbation of PD or improve the status of periodontal symptoms even during pregnancy.

Since the outcome is based on self-assessment of participants, psychological effects must be taken into consideration in evaluating the outcome of the intervention. We conclude that the intervention has produced a substantial effect on the basis of the following facts.

1) The SAPD score should be objective to a considerable extent because it has been proved to be efficient for screening PD [10].

2) The positive outcome at T3 was associated with decrease of the (log-transformed) proportion of the count of Candida species. Vieira Colombo [17] reported an association of a most common Candida species, C. albicans with PD.

3) The effect of intervention did not emerge at T2 but at T3. It must have been detected already at T2 if statistically significant psychological effects existed.

4) The probability of positive outcome at T3 increased with not only brushing proficiency but also use of mouthrinse at T1 and the regular scaling before pregnancy, hence the outcome is likely to reflect substantial effects.

\subsection{Oral Hygiene Education}

Affirmative rating of sufficiency of oral health education was a statistically significant predictor in LRA of positive outcome at T2. It has been reported that higher oral health literacy, or better knowledge of oral hygiene and behavior, is associated with better oral health condition of PD patients [18] and college students [19].

There seems to be a problem with oral health literacy in Japanese pregnant women. Although they can receive dental checkup free of charge once during pregnancy, the rate of visits in 2012 was as low as 23.4\% (253, 008/1080, 193) when calculated from the statistics in the annual report of the Ministry of Health, Labor and Welfare. Jiang et al. [20] reported that the frequency of dental visits in Minnesota was lower during pregnancy than before and after pregnancy with statistical significance. The reasons for decreased visits have been attributed to misunderstanding about its safety and appropriateness [21] as well as lower priority and financial concerns [21] [22].

It is necessary to promote the literacy of oral health of pregnant women and encourage them to receive dental checkup during pregnancy. 


\subsection{Use of Mouthrinse}

Use of mouthrinse at T1 was a statistically significant predictor of outcome at T3. Mouthrinse has been known to reduce dental plaque and improve gingivitis regardless whether its agent is chlorhexidine [23] or essential oil [24]. An RCT with pregnant women with PD proved that the use of antibacterial rinse containing cetylpyridinium chloride improved PD in pregnancy [25].

As tooth brushing is usually uneasy around T1 because of morning sickness, use of mouthrinse then would have led to the positive outcome at T3 by reducing dental plaque and preventing exacerbation of gingivitis.

\subsection{Regular Scaling}

The regular scaling before pregnancy was a statistically significant predictor in the logistic regression of positive outcome at both T2 and T3. Scaling by dental professionals reduces dental plaque and improves gingival inflammation, and the effects are enhanced by combining it with an oral education [26] [27]. Participants who had received regular scaling might have had better knowledge and behaviors for own oral health. Sambunjak et al. [28] reported that flossing combined with tooth brushing was effective for improving gingivitis. The frequency of scaling before pregnancy in this study was correlated with the frequency of flossing at T0 ( $\mathrm{r}=0.268, p<$ $0.001)$, $\mathrm{T} 1(\mathrm{r}=0.214, p<0.002)$, and T2 $(\mathrm{r}=0.251, p=0.002)$. This seems to indicate that participants who had regularly received scaling before pregnancy were more knowledgeable about oral health and tended to practice health behaviors such as flossing than others, which may have led to a positive outcome.

\subsection{Association of Microbiota with the Outcome}

The positive outcome at T3 was associated with decrease of the log-transformed proportion of Candida counts. This finding is in accordance with the report of Vieira Colombo et al. [17] which indicated the association of Candida albicans with periodontal inflammation and tissue destruction.

\subsection{Scope and Limitation}

The results of LRAs indicate that the brushing proficiency was more decisive (OR $=24.93)$ than assignment to the intervention group (OR = 3.83) for the outcome. Further study is needed to identify the factors affecting acquisition of brushing proficiency during pregnancy and improve the intervention program.

\section{Conclusions}

The effect of a health education program in early pregnancy including an oral health care education using a DVD displayer and training of the toothpick brushing method was evaluated by SAPD score and microbial data.

The LRA proved that the intervention $(\mathrm{OR}=3.83)$ and brushing proficiency $(\mathrm{OR}=24.93)$ were statistically significant predictors of the outcome at T3.

Thus an oral health education in early pregnancy followed by plaque checks in later pregnancy will improve pregnant women's oral health and the effect increases with their proficiency of toothpick method brushing.

The examination of microbial data suggests a significant association of existence or count of Candida species with the periodontal symptoms and the efficiency of toothpick method brushing to improve the status of periodontal symptoms.

\section{Acknowledgements}

This study was supported by JSPS Grant in Aid for Scientific Research (B) Number 18390596. We express our sincere gratitude to the participants and the people who enabled the research. We also thank Drs. Yuko Iwamoto and Fuminori Kato for their technical assistance and Dr. Anne J. Davis for correcting language use in the final version of the manuscript.

\section{Conflict of Interest}

The authors declare that no conflict of interest exists concerning this study. 


\section{References}

[1] Wu, M., Chen, S. and Jiang, S. (2015) Relationship between Gingival Inflammation and Pregnancy. Mediators of Inflammation, 2015, Article ID: 623472. http://dx.doi.org/10.1155/2015/623427

[2] Vogt, M., Sallum, A., Cecatti, J. and Morais, S. (2012) Factors Associated with the Prevalence of Periodontal Disease in Low-risk Pregnant Women. Reproductive Health, 9, No. 3. http://dx.doi.org/10.1186/1742-4755-9-3

[3] American College of Obstetricians and Gynecologists, Committee on Health Care for Underserved Women (2013) Committee Opinion No. 569: Oral Health Care during Pregnancy and through the Lifespan. American Journal of Obstetrics \& Gynecology, 122, 417-422.

http://www.acog.org/Resources-And-Publications/Committee-Opinions/Committee-on-Health-Care-for-UnderservedWomen/Oral-Health-Care-During-Pregnancy-and-Through-the-Lifespan http://dx.doi.org/10.1097/01.AOG.0000433007.16843.10

[4] Hashim, R. (2012) Self-Reported Oral Health, Oral Hygiene Habits and Dental Service Utilization among Pregnant Women in United Arab Emirates. International Journal of Dental Hygiene, 10, 142-146. http://dx.doi.org/10.1111/j.1601-5037.2011.00531.x

[5] Offenbacher, S., Katz, V., Fertik, G., Collins, J., Boyd, D., et al. (1996) Periodontal Infection as a Possible Risk Factor for Preterm Low Birth Weight. Journal of Periodontology, 67, 1103-1113. http://dx.doi.org/10.1902/jop.1996.67.10s.1103

[6] Jeffcoat, M., Geurs, N., Reddy, M., Cliver, S., Goldenberg, R. and Hauth, J. (2001) Periodontal Infection and Preterm Birth: Results of a Prospective Study. Journal of the American Dental Association, 132, 875-880. http://dx.doi.org/10.14219/jada.archive.2001.0299

[7] Chambrone, L., Guglielmetti, M., Pannuti, C. and Chambrone, L. (2011) Evidence Grade Associating Periodontitis to Preterm Birth and/or Low Birth Weight: I. A Systematic Review of Prospective Cohort Studies. Journal of Clinical Periodontology, 38, 795-808. http://dx.doi.org/10.1111/j.1600-051X.2011.01755.x

[8] Polyzos, N., Polyzos, I., Zavos, A., Valachis, A., Mauri, D., et al. (2010) Obstetric Outcomes after Treatment of Periodontal Disease during Pregnancy: Systematic Review and Meta-Analysis. BMJ, 341, Article ID: c7017. http://dx.doi.org/10.1136/bmj.c7017

[9] Uppal, A., Uppal, S., Pinto, A., Dutta, M., Shrivatsa, S., et al. (2010) The Effectiveness of Periodontal Disease Treatment during Pregnancy in Reducing the Risk of Experiencing Preterm Birth and Low Birth Weight: A Meta-analysis. Journal of the American Dental Association, 141, 1423-1434. http://dx.doi.org/10.14219/jada.archive.2010.0104

[10] Nakamura, G., Tsutsui, A., Horiguchi, I. and Turumoto, A. (1999) The Evaluation of Reliability and Validity on a Questionnaire, FSPD-34 Type, for Periodontal Disease (1). Journal of Dental Health, 49, 310-317. (In Japanese with English Abstract)

[11] Nakagawa, Y. and Daibo, I. (1985) Japanese Version of General Health Questionnaire-28. Nihon Bunka Kagakusha, Tokyo. (In Japanese)

[12] Fujiwara, N., Tsuruda, K., Iwamoto, Y., Kato, F., Odaki, T., et al. (2015) Significant Increase of Oral Bacteria in the Early Pregnancy Period in Japanese Women. Journal of Investigative and Clinical Dentistry. http://dx.doi.org/10.1111/jicd.12189

[13] Morita, M., Nishi, K. and Watanabe, T. (1998) Comparison of 2 Toothbrushing Methods for Efficacy in Supragingival Plaque Removal. The Toothpick Method and the Bass Method. Journal of Clinical Periodontology, 25, 829-831. http://dx.doi.org/10.1111/j.1600-051X.1998.tb02377.x

[14] Tsuneishi, M., Yamamoto, T. and Hirata, Y. (2010) Effects of Professional Toothbrushing by the Toothpick Method on Oral Malodor. Bulletin of Kanagawa Dental College, 38, 3-10.

[15] Mun, S., Chung, W., Min, S., Park, J., Kim, C., et al. (2014) Reduction in Dental Plaque in Patients with Mental Disorders through the Dental Hygiene Care Programme. International Journal of Dental Hygiene, 12, 133-1140. http://dx.doi.org/10.1111/idh.12053

[16] Filogônio, C., Soares, R., Horta, M., Penido, C. and Cruz, R. (2011) Effect of Vegetable Oil (Brazil Nut Oil) and Mineral Oil (Liquid Petrolatum) on Dental Biofilm Control. Brazilian Oral Research, 25, 556-561. http://dx.doi.org/10.1590/S1806-83242011000600014

[17] Vieira Colombo, A., Magalhães, C., Hartenbach, F., Martins do Souto, R. and Maciel da Silva-Boghossian, C. (2015) Periodontal-Disease-Associated Biofilm: A Reservoir for Pathogens of Medical Importance. Microbial Pathogenesis. http://dx.doi.org/10.1016/j.micpath.2015.09.009

[18] Wehmeyer, M., Corwin, C., Guthmiller, J. and Lee, J. (2014) The Impact of Oral Health Literacy on Periodontal Health Status. Journal of Public Health Dentistry, 74, 80-87. http://dx.doi.org/10.1111/j.1752-7325.2012.00375.x 
[19] Kanupuru, K., Fareed, N. and Sudhir, K. (2015) Relationship between Oral Health Literacy and Oral Health Status among College Students. Oral Health \& Preventive Dentistry, 13, 323-330.

[20] Jiang, P., Bargman, E., Garrett, N., Devries, A., Springman, S. and Riggs, S. (2008) A Comparison of Dental Service Use among Commercially Insured Women in Minnesota Before, during and after Pregnancy. Journal of the American Dental Association, 139, 1173-1180. http://dx.doi.org/10.14219/jada.archive.2008.0332

[21] Detman, L., Cottrell, B. and Denis-Luque, M. (2010) Exploring Dental Care Misconceptions and Barriers in Pregnancy. Birth, 37, 318-324. http://dx.doi.org/10.1111/j.1523-536X.2010.00427.x

[22] Le, M., Riedy, C., Weinstein, P. and Milgrom, P. (2009) Barriers to Utilization of Dental Services during Pregnancy: A Qualitative Analysis. Journal of Dentistry for Children, 76, 46-52.

[23] Van Strydonck, D., Slot, D., Van der Velden, U. and Van der Weijden, F. (2012) Effect of a Chlorhexidine Mouthrinse on Plaque, Gingival Inflammation and Staining in Gingivitis Patients: A Systematic Review. Journal of Clinical Periodontology, 39, 1042-1155. http://dx.doi.org/10.1111/j.1600-051X.2012.01883.x

[24] Stoeken, J., Paraskevas, S. and van der Weijden, G. (2007) The Long-Term Effect of a Mouthrinse Containing Essential Oils on Dental Plaque and Gingivitis: A Systematic Review. Journal of Periodontology, 78, 1218-1228. http://dx.doi.org/10.1902/jop.2007.060269

[25] Jiang, H., Xiong, X., Buekens, P., Su, Y. and Qian, X. (2015) Use of Mouth Rinse during Pregnancy to Improve Birth and Neonatal Outcomes: A Randomized Controlled Trial. BMC Pregnancy Childbirth, 15, 311. http://dx.doi.org/10.1186/s12884-015-0761-3

[26] Needleman, I., Nibali, L. and Di Iorio, A. (2015) Professional Mechanical Plaque Removal for Prevention of Periodontal Diseases in Adults-Systematic Review Update. Journal of Clinical Periodontology, 42, S12-S35. http://dx.doi.org/10.1111/jcpe.12341

[27] Chapple, I. L., Van der Weijden, F., Doerfe, C., Herrera, D., Shapira, L., et al. (2015) Primary Prevention of Periodontitis: Managing Gingivitis. Journal of Clinical Periodontology, 42, S71-S76. http://dx.doi.org/10.1111/jcpe.12366

[28] Sambunjak, D., Nickerson, J., Poklepovic, T., Johnson, T., Imai, P., et al. (2011) Flossing for the Management of Periodontal Diseases and Dental Caries in Adults. The Cochrane Database of Systematic Reviews, 12. 